\title{
The analytic nodal diffusion solver ANDES in multigroups for 3D rectangular geometry: Development and performance analysis
}

\author{
Juan-Andrés Lozano , Nuria García-Herranz, Carol Ahnert, José-María Aragonés \\ Departamento de Ingeniería Nuclear, Universidad Politécnica de Madrid (UPM), ETS Ingenieros Industriales, José G. Abascal, 2, 28006 Madrid, Spain
}

\begin{abstract}
A B S T R A C T
In this work we address the development and implementation of the analytic coarse-mesh finite-difference (ACMFD) method in a nodal neutron diffusion solver called ANDES. The first version of the solver is implemented in any number of neutron energy groups, and in 3D Cartesian geometries; thus it mainly addresses PWR and BWR core simulations.

The details about the generalization to multigroups and 3D, as well as the implementation of the method are given. The transverse integration procedure is the scheme chosen to extend the ACMFD formulation to multidimensional problems. The role of the transverse leakage treatment in the accuracy of the nodal solutions is analyzed in detail: the involved assumptions, the limitations of the method in terms of nodal width, the alternative approaches to implement the transverse leakage terms in nodal methods - implicit or explicit -, and the error assessment due to transverse integration. A new approach for solving the control rod "cusping" problem, based on the direct application of the ACMFD method, is also developed and implemented in ANDES.

The solver architecture turns ANDES into an user-friendly, modular and easily linkable tool, as required to be integrated into common software platforms for multi-scale and multi-physics simulations. ANDES can be used either as a stand-alone nodal code or as a solver to accelerate the convergence of whole core pin-by-pin code systems. The verification and performance of the solver are demonstrated using both proof-of-principle test cases and well-referenced international benchmarks.
\end{abstract}

\section{Introduction}

More accurate modeling of physical phenomena involved in present and future nuclear reactors requires a multi-scale and multi-physics approach. This challenge can be accomplished by the coupling of best-estimate core-physics, thermal-hydraulics and multi-physics solvers. In order to make viable that coupling, the current trends in reactor simulations are along the development of a new generation of tools based on user-friendly, modular, easily linkable, faster and more accurate codes to be integrated in common platforms. These premises are in the origin of the NURESIM Integrated Project within the 6th European Framework Program, which is envisaged to provide the initial step towards a Common European Standard Software Platform for nuclear reactors simulations (Cacuci et al., 2006). In the frame of this project and to reach the above-mentioned goals, a 3D multigroup nodal solver for neutron diffusion calculations called ANDES (Analytic Nodal Diffusion Equation Solver) has been developed and tested in-depth. ANDES can be used either as a standalone nodal code or as a solver to accelerate the convergence of whole core pin-by-pin code systems. In fact, it has already been implemented in our 3D lattice-core scope COBAYA3 code (Herrero et al., 2007) and as an alternative solver in the French CEA new code DESCARTES (Calvin, 2005).

ANDES solves the neutron diffusion equations in Cartesian 3D geometry and any number of groups utilizing the analytic coarse-mesh finite-difference (ACMFD) scheme to yield the nodal coupling equations. The ACMFD was formally introduced by Chao $(1999,2000)$ for the one-group one-dimensional diffusion equation, outlining the generalization to multigroups and multidimensional problems. In a first work, we demonstrated the efficiency of the ACMFD method in two-groups for 2D nodal Cartesian geometry (García-Herranz et al., 2003). Recently, we established the full mathematical basis for the multidimensional cases with more than two energy groups, where complex rather than real matrices have to be dealt with (Aragonés et al., 2007).

This ACMFD scheme comes out explicitly from the analytic solution of the diffusion equations, with no approximation in 1D. In multigroup problems, it lies in the transformation of the physical space of group fluxes into the modal space of the complete base of eigenvectors of the multigroup diffusion equation matrix. The resulting ACMFD coupling equations are matrix-vector relations and, in this sense, it can be considered as a highly accurate scheme, because it includes in the nodal solution the effects of the intranodal flux shape and spectral variation. 
The state-of-the-art nodal codes use polynomial or analytical flux expansions to solve the multidimensional diffusion equations within homogeneous nodes. Most of them use the nodal expansion method (NEM) or the analytical nodal method (ANM) accelerated by a nonlinear CMFD iterative solution scheme (Finnemann et al., 1977; Smith, 1986; Turinsky et al., 1994; Joo et al., 1998). The 1D transverse-integrated two-node problems are solved using the NEM or ANM methods, and the computed interface currents are used to update the nonlinear coupling factors included in the modified finite-difference scheme. These coupling factors are scalar group-by-group approximates, which constitutes the main difference regarding the ACMFD coupling relations. Other nodal methods have also used the diagonalization of the multigroup diffusion equations, such as the multigroup analytic nodal method (MANM), Vogel and Weiss (1992), Müller and Wiederhold, (1995) and the analytic function expansion nodal (AFEN), which uses a truncated flux expansion of 3D analytic basis functions, without transverse integration (Noh and Cho, 1994).

This paper completes the developments for the generalization of the ACMFD method to multidimensional problems, addressing its implementation in a nodal solver of the multigroup diffusion equations in $3 \mathrm{D}$ rectangular geometry. The paper consists of six sections. In Section 2, we analyze the particularities of the ACMFD methodology in multigroups and multidimensions in Cartesian geometry; efforts have been focused towards minimizing errors due to the transverse leakage treatment and thus refining the accuracy level of the methodology. In Section 3, a new approach for solving the control rod "cusping" problem - found when control rods are partially inserted in the nodes -, based on a direct application of the ACMFD method, is developed and verified. In Section 4, we focus on the design of the solver ANDES, explaining the data flux management and the numerical iterative procedure. Section 5 is devoted to numerical results to carry out a consistent verification of the solver, where different benchmark tests have been selected, each one appropriate to show the performance of each different model involved in ANDES. Finally, conclusions are presented in Section 6 .

\section{The analytic multigroup diffusion theory for multidimensional problems}

The full basis of the methodology is given in Aragonés et al. (2007). Here we revisit the solution in multidimensional diffusion cases in order to point out the role of the transverse leakage treatment in obtaining an accurate nodal solution.

\subsection{Solution of the modal equations in $1 D$ with external sources}

In 1D Cartesian geometry with homogeneous nodes, the $G$ uncoupled modal equations obtained by transformation of the $G$ multigroup diffusion equations can be written as follows:

$$
\begin{aligned}
\frac{\mathrm{d}^{2}\left|\psi_{m}(x)\right\rangle}{\mathrm{d} x^{2}}-\lambda_{m}\left|\psi_{m}(x)\right\rangle & =-\left|S_{m}(x)\right\rangle=-\boldsymbol{R D}^{-1}\left|S_{g}(x)\right\rangle ; \quad m \\
& =1, G
\end{aligned}
$$

where $\lambda_{m}$ are the eigenvalues of the matrix of the multigroup equations, $\Psi_{m}(x)$ the modal fluxes, $\boldsymbol{R}^{-1}$ the matrix of eigenvectors, $\boldsymbol{D}$ the diagonal matrix of the diffusion coefficients per energy group, $S_{g}(x)$ a space-dependent external source per energy group and $s_{m}(x)$ the corresponding modal source (Aragonés et al., 2007). Let us suppose that the distribution of the external source is analytically known. Then, Eq. (1) has an analytical solution given by the solution of the homogeneous equation plus a particular solution $p_{m}(x)$ :
$\psi_{m}(x)=A_{m} \mathrm{e}^{+\alpha_{m} x}+B_{m} \mathrm{e}^{-\alpha_{m} x}+p_{m}(x)$

$\frac{\mathrm{d}^{2}}{\mathrm{~d} x^{2}} p_{m}(x)-\lambda_{m} p_{m}(x)=-s_{m}(x) ; \quad \alpha_{m}=\sqrt{\lambda_{m}}$

$A_{m}$ and $B_{m}$ are constants to be determined for a given node and face $(x= \pm H / 2)$ by imposing the following two conditions: the modal flux and current at that node interface. Then, substituting in the nodeaverage modal flux, the ACMFD modal relation is obtained:

$$
\begin{aligned}
\psi_{m} & \left(\mp \frac{H}{2}\right)-p_{m}\left(\mp \frac{H}{2}\right) \\
& =C_{m}^{f}\left[\bar{\psi}_{m}-\bar{p}_{m}\right] \pm C_{m}^{j} \frac{H}{2}\left[J_{m}\left(\mp \frac{H}{2}\right)+p_{m}^{\prime}\left(\mp \frac{H}{2}\right)\right]
\end{aligned}
$$

$C_{m}^{f}$ and $C_{m}^{j}$ are scalar modal coefficients only dependent on both the eigenvalue and the nodal length $H$, which are thus given by:

$C_{m}^{f}=\frac{2 \alpha_{m} H}{\mathrm{e}^{+\alpha_{m} H}-\mathrm{e}^{-\alpha_{m} H}} ; \quad C_{m}^{j}=\frac{\mathrm{e}^{+\alpha_{m} H}+\mathrm{e}^{-\alpha_{m} H}-2}{\mathrm{e}^{+\alpha_{m} H}-\mathrm{e}^{-\alpha_{m} H}} \frac{2}{\alpha_{m} H}$

All terms in Eq. (3) related to the particular solution can be grouped together into a modal term $T_{m}$, called external-source term:

$T_{m}\left(\mp \frac{H}{2}\right)=C_{m}^{f} \bar{p}_{m}-p_{m}\left(\mp \frac{H}{2}\right) \mp \frac{H}{2} C_{m}^{j} p_{m}^{\prime}\left(\mp \frac{H}{2}\right)$

Finally, transforming these scalar relations in the modal space to the physical space of the group fluxes, we obtain the ACMFD formula for each left and right interface:

$\left|\phi_{g}\left(\mp \frac{H}{2}\right)\right\rangle=\boldsymbol{A}^{f}\left|\bar{\phi}_{g}\right\rangle \pm \frac{H}{2} \boldsymbol{A}^{j} \boldsymbol{D}_{g}^{-1}\left|J_{g}\left(\mp \frac{H}{2}\right)\right\rangle-R^{-1}\left|T_{m}\left(\mp \frac{H}{2}\right)\right\rangle$

$A^{f}=R^{-1} C^{f} R ; \quad A^{j}=R^{-1} C^{j} R$

where $\boldsymbol{C}^{d}$ and $\boldsymbol{C}^{j}$ are now the diagonal matrices of the scalar modal coefficients. Eq. (6) is a matrix relation that is exact in the 1D multigroup diffusion approximation for homogeneous nodes with a known space-dependent external source.

From (6), following Chao (Chao, 2000) and assuming $\boldsymbol{A}^{\boldsymbol{f} *}\left|\bar{\phi}_{g}\right\rangle=\boldsymbol{A}^{\mathbf{f}}\left|\bar{\phi}_{g}\right\rangle-R^{-1}\left|T_{\boldsymbol{m}}\left(\mp \frac{H}{2}\right)\right\rangle$, the (column) vector of multigroup net currents at the interfaces is obtained as a function of the vectors of node-average and interface fluxes by:

$\left|J_{g}\left(\mp \frac{H}{2}\right)\right\rangle=\mp \boldsymbol{D} \boldsymbol{A}^{j-1} \frac{\boldsymbol{A}^{\mathbf{f}_{*}}\left|\bar{\phi}_{g}\right\rangle-\left|\bar{\phi}_{g}\left(\mp \frac{H}{2}\right)\right\rangle}{H / 2}$

Eq. (8) are the half-node ACMFD formula, for each left and right node interface; which are much alike the linear finite-difference discretization of the diffusion approximation (Fick's law), but including the coarse-mesh and spectral effects of the analytic intra-nodal flux variation in space and spectra, which is "exact" in the 1D multigroup diffusion approximation assumed above. The matrix $A^{f^{*}}$ "corrects" the vector node average fluxes in the "gradient" term of diffusion Fick's law and the matrix $\left[\boldsymbol{A}^{j}\right]^{-\mathbf{1}}$ "corrects" the diffusion coefficient diagonal matrix $D$. But the general ACMFD Fick's law (8) is not anymore just a "corrected" or non-linear synthetic scalar relation within each group, but matrix-vector products coupling all groups at the node interfaces. Only if $H \rightarrow 0$ (finemesh), since then the matrices $\boldsymbol{A}^{\boldsymbol{f}}$ and $\boldsymbol{A}^{\boldsymbol{j}}$ become the identity matrix, we recover the original (scalar) Fick's law.

\subsection{Solution of the modal equations in $3 D$}

In multidimensional problems the modal Eq. (1) can be written as

$\nabla^{2}\left|\psi_{m}(\boldsymbol{r})\right\rangle-\left[\lambda_{m}\right]\left|\psi_{m}(\boldsymbol{r})\right\rangle=-\left|s_{m}(\boldsymbol{r})\right\rangle ; \quad m=1, G$

To obtain the ACMFD modal relation, a transverse flux integration scheme can be applied. Eq. (9) is then transverse integrated (i.e. 
in $y$ and $z$ ) over the node and averaged over the transverse volumes to yield the reduced $1 \mathrm{D}$ modal equations (i.e. in $x$ ):

$\frac{\mathrm{d}^{2}\left|\psi_{m}(x)\right\rangle}{\mathrm{d} x^{2}}-\lambda_{m}\left|\psi_{m}(x)\right\rangle=-\boldsymbol{R D}^{-1}\left|S_{g}^{*}(x)\right\rangle$

Eq. (10) is identical to Eq. (1), but now the external source $S_{\sigma}^{*}(x)$ includes the transverse leakage $L_{g}(x)$ as an additional term. In Cartesian 3D problems:

$$
\begin{aligned}
& S_{g}^{*}(x)=S_{g}(x)-L_{y}(x)-L_{z}(x)=S_{g}(x)-L_{g}(x) \\
& \left.L_{y}(x)=\left[J_{y}^{+H_{y} / 2}(x)-J_{y}^{-H_{y} / 2}(x)\right] / H_{y} ; \quad L_{z}(x)=J_{z}^{+H_{z} / 2}(x)-J_{z}^{-H_{z} / 2}(x)\right] / H_{z}
\end{aligned}
$$

As can be seen, the external source depends on the distribution of the transverse leakage in the node interfaces parallel to $x$-axis. $\mathrm{Gi}$ ven that in a nodal diffusion solver only the net currents at the node interfaces are available during the iterative process, the transverse leakage distribution is unknown. Consequently, the analytical solution of Eq. (10) will not match completely the true 3D solution, as occurred in Eq. (1) for the $1 \mathrm{D}$ case.

The most appropriate option in nodal solvers to infer the transverse leakage profile is to interpolate, from the previous nodal solution, the transverse net currents along the lines or planes of node interfaces, by a nonlinear iterative scheme. In consequence, an error in the particular solution of Eq. (2) is introduced, being transmitted to the external-source term $T_{m}$ (or transverse-leakage term) and then to the final ACMFD relation (6). $T_{m}$ has then an important role in obtaining an accurate solution as it is going to gather all the error associated to the transverse leakage interpolation.

\subsubsection{Transverse leakage interpolation}

In ANDES, following earlier transverse leakage approximations (Finnemann et al., 1977; Smith, 1979), a polynomial interpolation, parabolic or cubic, of the transverse net currents is assumed, due to the low computational cost and the easiness of the analytic solution of equations. Using Eq. (2) and considering a cubic fit for the transverse leakage term: $-\left|\boldsymbol{s}_{\mathrm{m}}(x)\right\rangle=\left|\boldsymbol{I}_{\mathrm{m}}(x)\right\rangle=\boldsymbol{R} \boldsymbol{D}^{-1}\left|\boldsymbol{L}_{\mathrm{g}}(x)\right\rangle$, we obtain the particular solution:

$p_{m}(x)=\frac{1}{-\alpha_{m}^{2}} l_{m}(x)+\frac{1}{-\alpha_{m}^{2}} \cdot \frac{l_{m}^{\prime \prime}(x)}{\alpha_{m}^{2}}$

From this, we obtain the face averaged value and the values and derivatives at each node corner or aristae: $\bar{p}_{m}, p_{m}\left( \pm \frac{H}{2}\right), p_{m}^{\prime}\left( \pm \frac{H}{2}\right)$. For $x=-H / 2$ (similarly for $x=+H / 2)$ :

$\bar{p}_{m}=\frac{1}{-\alpha_{m}^{2}} \bar{l}_{m}+\frac{1}{-\alpha_{m}^{2}} \cdot \frac{1}{\alpha_{m}^{2}}\left(l_{m}^{\prime \prime \prime}\left(-\frac{H}{2}\right)+l_{m}^{\prime \prime}\left(-\frac{H}{2}\right) \cdot \frac{H}{2}\right)$

$p_{m}\left(-\frac{H}{2}\right)=\frac{1}{-\alpha_{m}^{2}} l_{m}\left(-\frac{H}{2}\right)+\frac{1}{-\alpha_{m}^{2}} \cdot \frac{l_{m}^{\prime \prime}\left(-\frac{H}{2}\right)}{\alpha_{m}^{2}}$

$p_{m}^{\prime}\left(-\frac{H}{2}\right)=\frac{1}{-\alpha_{m}^{2}} l_{m}^{\prime}\left(-\frac{H}{2}\right)+\frac{1}{-\alpha_{m}^{2}} \cdot \frac{l_{m}^{\prime \prime \prime}\left(-\frac{H}{2}\right)}{\alpha_{m}^{2}}$

By substitution of these values into Eq. (5), and taking into account that the modal transverse leakage at the (corner) left side $l_{m}(-\mathrm{H} / 2)$ can be written in terms of the interface averaged transverse leakage value $\bar{l}_{m}$ and the derivatives at the corner $l_{m}^{\prime}, I_{m}^{\prime \prime}, l_{m}^{\prime \prime \prime}$, we obtain the following relation for the transverse leakage term:

$$
\begin{aligned}
T_{m}\left(-\frac{H}{2}\right)= & \bar{l}_{m}\left(\frac{C_{m}^{f}-1}{-\alpha_{m}^{2}}\right)-\frac{H}{2} l_{m}^{\prime}\left(-\frac{H}{2}\right)\left(\frac{C_{m}^{j}-1}{-\alpha_{m}^{2}}\right) \\
& +\frac{l_{m}^{\prime \prime}\left(-\frac{H}{2}\right)}{\alpha_{m}^{2}}\left(\frac{C_{m}^{f}-1}{-\alpha_{m}^{2}}-\frac{H^{2}}{6}\right)+\frac{H}{2} \frac{l_{m}^{\prime \prime \prime}}{\alpha^{2}}\left(\frac{C_{m}^{f}-C_{m}^{j}}{-\alpha_{m}^{2}}-\frac{H^{2}}{12}\right)
\end{aligned}
$$

A singularity appears in (16) as the eigenvalue $\alpha_{m}^{2}$ approaches zero $\left(\alpha_{m}^{2} \rightarrow 0\right)$. However by a truncated Taylor series expansion of the modal coefficients $C_{m}^{f}$ and $C_{m}^{j}$ given by Eq. (4), it can be demonstrated that $T_{m}$ reduces to a finite value as $\alpha_{m}^{2}$ gets closer to zero. To avoid the singularity, that limit is taken for the nodes in the neighborhood of zero eigenvalue during nodal iterations.

\subsubsection{Errors due to the transverse leakage interpolation}

Following, an analytical study on the errors introduced by the transverse integration is performed. The transverse leakage profile can be assumed as a piecewise constant function with $N$ step discontinuities. In this case, the ACMFD relation remains unchanged, being the transverse leakage term:

$$
\begin{aligned}
T_{m}\left(-\frac{H}{2}\right)= & \bar{l}_{m}\left(\frac{C_{m}^{f}}{-\alpha_{m}^{2}}\right)-l_{m}\left(-\frac{H}{2}\right)\left(\frac{1}{-\alpha_{m}^{2}}\right) \\
& -\frac{1}{-\alpha_{m}^{2}} \sum_{i=1}^{N} C_{m}^{f \Lambda}\left(x_{i}\right) \cdot \Delta l_{m}^{i}
\end{aligned}
$$

where $\Delta l_{m}^{i}$ is the transverse leakage step for the coordinate $x_{i}$ $\in\left(-\frac{H}{2}, \frac{H}{2}\right)$ and

$C_{m}^{f \Delta}\left(x_{i}\right)=\frac{\mathrm{e}^{\alpha_{m}\left(H / 2-x_{i}\right)}-\mathrm{e}^{-\alpha_{m}\left(H / 2-x_{i}\right)}}{\mathrm{e}^{\alpha_{m} H}-\mathrm{e}^{-\alpha_{m} H}}$

This function approaches 1 as $x_{i}$ approaches $-H / 2$, and it approaches 0 as $x_{i}$ approaches the opposite face, $+H / 2$. Consequently, the transverse leakage steps close to the considered interface have more influence on the modal flux.

The most important key of the step function is that it can be used to approximate any transverse leakage profile. In the limit of differential steps, Eq. (17) is written as

$T_{m}\left(-\frac{H}{2}\right)=\bar{l}_{m}\left(\frac{C_{m}^{f}}{-\alpha_{m}^{2}}\right)+\frac{1}{-\alpha_{m}^{2}} \int_{-H / 2}^{H / 2} \frac{\mathrm{d}\left(C_{m}^{f \Delta}(x)\right)}{\mathrm{d} x} \cdot I_{m}(x) \cdot \mathrm{d} x$

This Eq. (19) can be applied to compute the modal flux at the left nodal interface taking the real transverse leakage profile $(R)$ and taking the interpolated profile $(I)$. Assuming that the nodal average flux and the average interface currents are the same in both cases, the interface modal flux difference is given by:

$$
\begin{aligned}
\varepsilon_{m} & =\psi_{m}^{I}\left(-\frac{H}{2}\right)-\psi_{m}^{R}\left(-\frac{H}{2}\right) \\
& =\frac{1}{\alpha_{m}^{2}} \int_{0}^{H} \frac{\mathrm{d}\left(C_{m}^{f \Delta}(x)\right)}{\mathrm{d} x} \cdot\left(l_{m}^{I}(x)-I_{m}^{R}(x)\right) \cdot \mathrm{d} x
\end{aligned}
$$

Eq. (20) shows that as the modal eigenvalue decreases, the error due to the transverse leakage interpolation becomes larger. The error depends also on the difference between the transverse leakage profiles weighted with a known eigenvalue-dependent function.

\subsubsection{Implicit or explicit transverse leakage in ACMFD method}

Once the transverse leakage modal term $T_{m}( \pm H / 2)$ has been calculated, it is introduced in the ACMFD expression. Two different approaches can be developed:

(1) An explicit approach, where the term $T_{m}( \pm H / 2)$ is considered as an external source. From the transverse net currents from the previous nodal solution, $T_{m}$ is computed and the ACMFD resulting expression is Eq. (6).

(2) An implicit way, where the term $T_{m}( \pm H / 2)$ is written as the product of an arbitrary matrix $\boldsymbol{B}^{f}$ (with $G \times G$ unknowns, it has to accomplish $G$ conditions and its shape must lead to a stable solution) by the vector of nodal average fluxes. The resulting ACMFD expression is the following (note that 
$\boldsymbol{A}^{f}$ is a matrix depending only on the node, while $\boldsymbol{A}^{\boldsymbol{f}^{*}}=\boldsymbol{A}$ $\boldsymbol{f}_{-\boldsymbol{B}} \boldsymbol{f}$ is a matrix depending moreover on the nodal interface):

$$
\begin{aligned}
\left|\phi_{g}\left(\mp \frac{H}{2}\right)\right\rangle= & \boldsymbol{A}^{f}\left|\bar{\phi}_{g}\right\rangle \pm \frac{H}{2} \boldsymbol{A}^{j} \boldsymbol{D}_{g}^{-1}\left|J_{g}\left(\mp \frac{H}{2}\right)\right\rangle-\boldsymbol{B}^{f}\left|\bar{\phi}_{\mathbf{g}}\right\rangle \\
& =\boldsymbol{A}^{\boldsymbol{f}^{*}}\left|\bar{\phi}_{\mathrm{g}}\right\rangle \pm \frac{H}{2} \boldsymbol{A}^{\boldsymbol{j}} \boldsymbol{D}_{g}^{-1}\left|J_{g}\left(\mp \frac{H}{2}\right)\right\rangle
\end{aligned}
$$

Both approaches are equivalent, yielding the same nodal solution and showing the same convergence rate. The implicit approach may improve the stability of the linear system resolution method, because in some cases it makes the matrix more diagonally dominant.

If we consider two adjacent nodes, and apply the continuity conditions of the physical fluxes and currents at the common interface, we obtain that the interface currents at this interface can be completely determined in terms of the average fluxes in all groups of the two nodes. Using the currents calculated this way to express the leakage term in the neutron balance relation in every node, we obtain the finite-difference multigroup diffusion equation system provided by the ACMFD method. It reduces to a minimum the number of unknowns, the average fluxes per node and group. In $3 \mathrm{D}$ this system is seven-block diagonal, with blocks of $G \times G$ nonzero elements, which is amenable to Krylov space solvers, such as GMRES.

\subsection{Limitations of the ACMFD formulation}

Paying attention to expression (4), the denominators of the modal coefficients $C_{m}^{f}$ and $C_{m}^{j}$ become zeros if the argument $\alpha_{m-}$ $H=$ in $\pi$. When $\alpha_{m} H=0$, the numerator also vanishes, and a Taylor series expansion allows to calculate the modal coefficients in this limit:

$\lim _{\alpha_{m} H \rightarrow 1} C_{m}^{f}=1, \quad \lim _{\alpha_{m} H \rightarrow 0} C_{m}^{j}=1$
To avoid $\alpha_{m} H=i \pi$ during the iterative process (which only can happen for the fundamental mode in supercritical nodes), given that $\alpha_{m}$ only depends on the nodal cross-sections and on the $k_{\text {eff }}$ value, two different conditions can be imposed:

(i) To initialize the outer iterations with large values of $k_{\text {eff }}$ (small initial values yields more negative fundamental eigenvalues $\alpha_{m}^{2}$ ).

(ii) To use a nodal size fine enough. No problems are found using a node size equal to the fuel assembly width of real cores. The nodal size must be smaller than the half wave length of the nodal analytic solutions, as widely discussed in (Ziyong et al., 2006).

\section{Control rod "cusping" correction using the ACMFD formulation}

When a control rod is partially inserted into a node, the intranodal flux distribution undergoes a strong distortion. If such intranodal flux distribution is not properly accounted for computing the homogenized nodal cross-sections, so called "cusping" effects appear in the differential rod reactivity worth when the insertion of a control rod varies.

A new approach to correct the rod "cusping" problem in 3D calculations is proposed here, as a result of the direct application of the ACMFD formulation. The proposed model has been implemented in the ANDES solver, and the verification calculations have confirmed the validity of the method.

Let us suppose a partially rodded node $(P)$ adjacent to a fully rodded node $(R)$ and to an unrodded node $(U)$. In ANDES, the partially rodded node $(P)$ is split into two parts: rodded (PR) and unrodded (PU) part, each assumed homogeneous (see Fig. 1). Applying the ACMFD relation (6) at the axial internal interface between the two parts of the node, and given the flux and current continuity at this interface, the inner axial current can be expressed as a function of the two average fluxes in each part, $\left|\bar{\phi}_{\mathrm{gPR}}\right\rangle$ and $\left|\bar{\phi}_{\mathrm{gPU}}\right\rangle$.

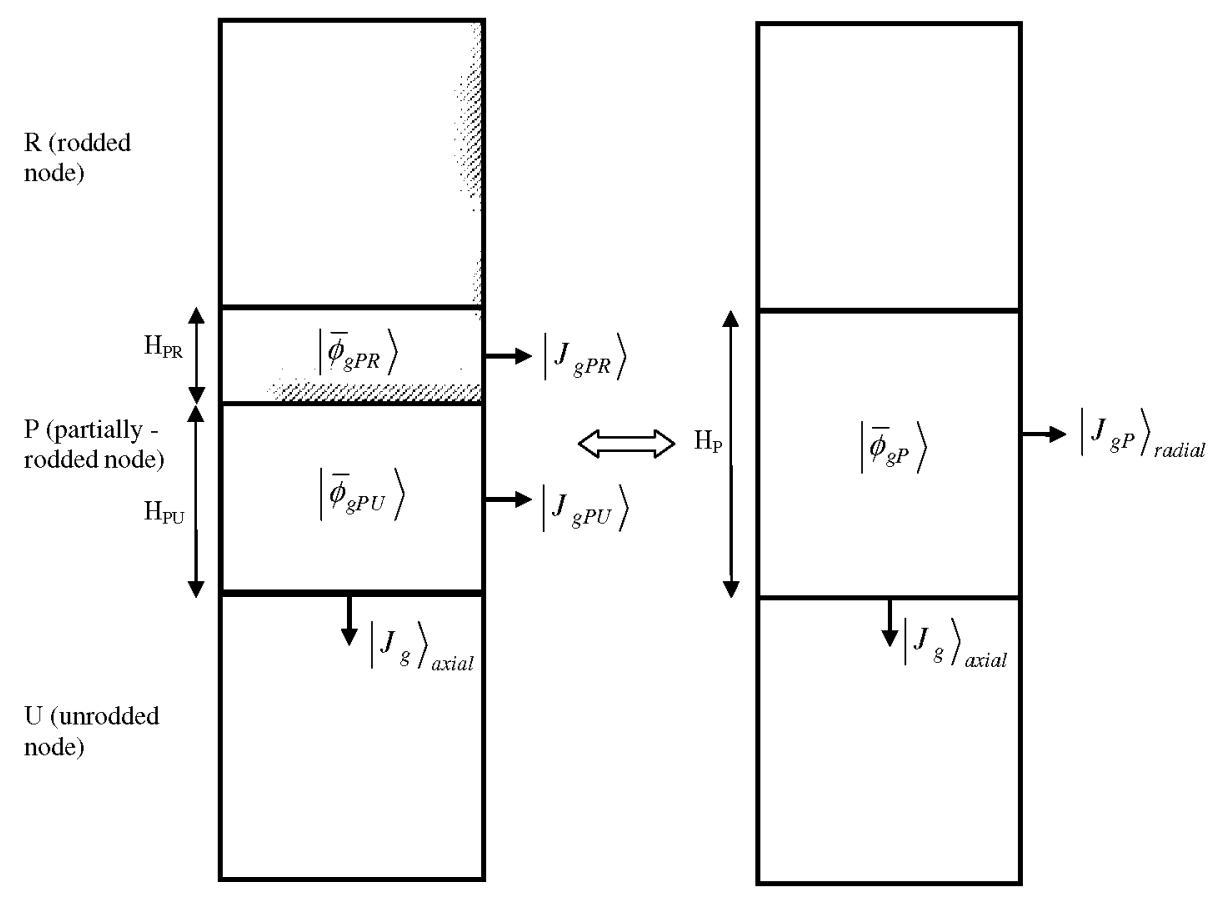

Fig. 1. Geometry and magnitudes involved in the control rod "cusping" correction. 
These average fluxes are determined by solving the neutron balance equation for the two parts of the node, called "one-partiallyrodded-node" problem. This is done by imposing the external surface average currents, available from the previous nodal solution, as boundary conditions. In the axial direction (upper and lower boundaries) they are known from the global calculation; however in the radial direction, the needed average currents at each partial interface, $\left.U_{g \mathrm{gR}}\right\rangle$ and $\left.U_{g \mathrm{Pu}}\right\rangle$, have to be interpolated from the radial average net current at the full-height node interface $\left\langle U_{g \mathrm{p}}\right\rangle_{\text {radial }}$ of the partially rodded node and its two axial neighbours, which is a common practice to address the rod cusping problem in nodal methods. We use two quadratic splines for the axial dependence of the radial currents. The six coefficients are determined imposing the following conditions: the radial averages at the three full nodes $(R),(P),(U)$; the continuity of the currents and their derivatives at the interface between (PR) and (PU); and the minimum square deviation of the currents at the interfaces between $(R)$ and (PR) and between (PU) and $(U)$. This approach is more based on empirical reasons than on theoretical foundations, but it is used because it provides good results as it adapts quite well to the physical current profile.

The average fluxes calculated from the one-partially-roddednode problem are inserted into the following equation to perform a volume weighting of the rodded and unrodded cross-sections. This way the homogenized nodal cross-sections preserve the reaction rates at the node.

$\bar{\Sigma}_{X, g}=\frac{\Sigma_{X, g}^{\mathrm{PU}} \bar{\phi}_{g}^{\mathrm{PU}} x_{V}^{\mathrm{PU}}+\Sigma_{X, g}^{\mathrm{PR}} \bar{\phi}_{g}^{\mathrm{PR}} \chi_{V}^{\mathrm{PR}}}{\bar{\phi}_{g}^{\mathrm{PU}} x_{V}^{\mathrm{PU}}+\bar{\phi}_{g}^{\mathrm{PR}} \chi_{V}^{\mathrm{PR}}}$

where $\bar{\phi}_{\mathrm{g}}^{\mathrm{PU}}, \bar{\phi}_{\mathrm{g}}^{\mathrm{PR}}$ are the average fluxes at the partially unrodded and rodded regions of the node; $\Sigma_{X, g}^{\mathrm{PU}}, \Sigma_{X, g}^{\mathrm{PR}}$ are the corresponding crosssections for reaction $X$ in the $g$ energy group and $x_{V}^{\mathrm{PU}}, x_{V}^{\mathrm{PR}}$ the corresponding volume fractions.

Direct application of this approach provides appropriate results for homogeneous nodes. However, for heterogeneous nodes, the flux-volume weighting of the homogenized cross-sections is not enough to eliminate the rod "cusping" effect. Just like in the application of the ACMFD method to actual operating reactors, where the heterogeneity of the fuel assemblies requires the use of flux heterogeneity factors at the radial $(x-y)$ nodal interfaces, to attenuate the axial rod "cusping" effect to an acceptable level, a pair of axial heterogeneity factors have to be introduced at both axial interfaces of the partially rodded node.

Consider a partially rodded node as shown in Fig. 1, and let us notice the lower nodal interface (between PU and $U$ ). Both the homogeneous and heterogeneous average flux at this interface can be computed using the ACMFD relation in Eq. (21) applied to the lower axial interface of the homogeneized node and of the unrodded part of the node, respectively:

$\left|\boldsymbol{\phi}_{\mathrm{gPU}}^{\mathrm{hom}}\right\rangle=\boldsymbol{A}^{f *}\left|\bar{\phi}_{g P}\right\rangle+\frac{H_{P}}{2} \boldsymbol{A}^{j} \boldsymbol{D}_{g}^{-1}\left|J_{g}\right\rangle_{\text {axial }}$
$\left|\boldsymbol{\phi}_{\mathrm{gPU}}^{\text {het }}\right\rangle=\boldsymbol{A}_{\text {het }}^{f_{*}}\left|\bar{\phi}_{\mathrm{gPU}}\right\rangle+\frac{H_{\mathrm{PU}}}{2} \boldsymbol{A}_{\text {het }}^{j} \boldsymbol{D}_{g}^{-1}\left|J_{g}\right\rangle_{\text {axial }}$

This gives us the way to compute the scalar (per group) flux heterogeneity factors at this lower axial interface (and similarly for the upper one):

$\phi_{\mathrm{gPU}}^{\mathrm{het}}=f_{\mathrm{gPU}} \cdot \phi_{\mathrm{gPU}}^{\mathrm{hom}} \rightarrow f_{\mathrm{gPU}}=\frac{\phi_{\mathrm{gPU}}^{\mathrm{het}}}{\phi_{\mathrm{gPU}}^{\mathrm{hom}}}$

The axial interface flux heterogeneity factors calculated this way will be used in the partially rodded nodes for the whole core calculation in the next iteration step on transverse leakage.

It should be noted that in this approach implemented in the $A N$ $D E S$ solver for the treatment of the rod "cusping" effect, the unique source of error introduced is due to the interpolation of the radial average currents. This error affects not only the neutron balance equation but also the transverse leakage profile in the ACMFD relation in the axial direction for the partially rodded nodes and their radial neighbours.

\section{Iterative scheme in ANDES}

The calculation flow in the ANDES solver for steady-state problems is shown in Fig. 2. Two types of steady-state problems can be solved: the eigenvalue and the fixed source problem. The solution of the eigenvalue problem is composed of three levels of iterations: the outer iteration over the $k_{\text {eff }}$ eigenvalue, the intermediate iteration over the transverse leakage (not strictly necessary since the transverse leakage is updated in the outer iteration loop), and the inner iteration in which the block-diagonal linear system is solved.

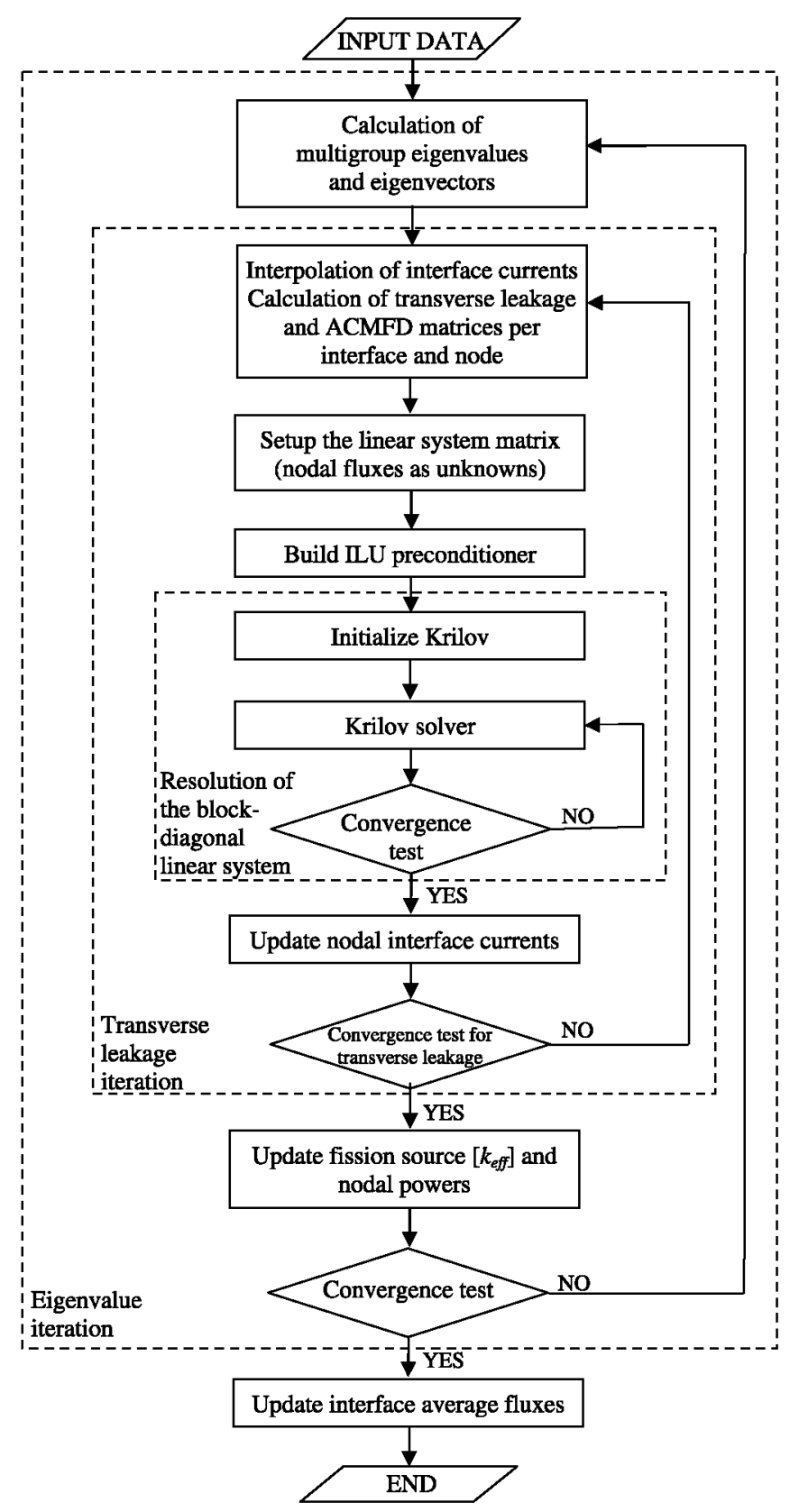

Fig. 2. Flow diagram for the eigenvalue calculation in ANDES. 
During the fixed source problem calculation, the outer $k_{\text {eff }}$ iterations are skipped, and the transverse leakage iteration loop has to be performed to update the transverse leakage, once the nodal average fluxes are available from the inner iteration solution process. Flat nodal fixed sources have been implemented, but other intranodal functional variations can be readily implemented as well.

In writing the solver, special attention has been paid to the data management in order to encapsulate all the ANDES interface variables (input and output). These features make it appropriate to be integrated in other code systems, with different data structure. In the framework of the NURESIM project, the solver has been integrated in the SALOME platform (Cacuci et al., 2006), where the coupling with any other code in the platform does not require to compile them together; instead, the coupling consists of a formatted data structure exchange. SALOME also provides a module for pre/post-processing (an example is shown in Fig. 7).

\section{Numerical results}

An extended set of benchmarks has been performed during the process of ANDES verification, ranging from proof-of -principle test problems, to evaluate the mesh discretization error and its convergence rate with mesh size, to full-core steady state well-established international benchmarks.

Table 1 shows the list of benchmarks and the most noteworthy results. Among all of them, two benchmarks have been selected to be analyzed here. The first one is a typical two-dimensional PWR color-set problem. This test case is designed to show the performance of the transverse leakage approximation in the ACMFD formulation. The second one is a full core benchmark (PWR MOX/UO2 core transient benchmark) to demonstrate the accuracy of the results and to analyze the computing time for a 3D solution with different number of energy groups (2,4 and 8 ). Additionally, we have designed a simplified mini-core test case in order to assess the rod "cusping" treatment in ANDES.

Table 1

Overview of ANDES results for the different benchmarks, with four nodes per assembly and two energy groups

\begin{tabular}{|c|c|c|c|c|c|}
\hline Test/Benchmark id. & Case & $\begin{array}{l}\text { Critical } \\
\text { Boron } \\
\text { conc. } \\
\left(\text { ppm) } / K_{\text {eff }}\right.\end{array}$ & $\begin{array}{l}\text { Error with } \\
\text { reference } \\
(\mathrm{ppm}) / \\
(\mathrm{pcm})\end{array}$ & FXY & $\begin{array}{l}\text { Relative } \\
\text { error } \\
\text { (FXY) } \\
(\%)\end{array}$ \\
\hline $\begin{array}{l}\text { 2D PWR color-set } \\
\text { benchmark }\end{array}$ & - & 0.958780 & 2.7 & 1.1781 & 0.02 \\
\hline \multirow{6}{*}{$\begin{array}{l}\text { LWR core transient } \\
\text { benchmarks } \\
\text { (Finnemann and } \\
\text { Galati, 1991) }\end{array}$} & $\mathrm{A} 1$ & 561.62 & 6.1 & 1.9109 & 0.10 \\
\hline & $\mathrm{A} 2$ & 1158.03 & 2.6 & 1.1997 & 0.14 \\
\hline & B1 & 1248.42 & 6.2 & 1.2760 & 0.00 \\
\hline & B2 & 1187.68 & 1.7 & 1.1723 & 0.20 \\
\hline & $\mathrm{C} 1$ & 1128.75 & 6.5 & 1.4440 & 0.07 \\
\hline & $\mathrm{C} 2$ & 1158.03 & 2.6 & 1.1997 & 0.14 \\
\hline \multirow{2}{*}{$\begin{array}{l}\text { PWR benchmark on } \\
\text { uncontrolled rods } \\
\text { withdrawal at zero } \\
\text { power(Fraikin, 1997) }\end{array}$} & A & 1262.4 & 0.3 & 1.243 & 0.08 \\
\hline & B & 793.3 & 0.3 & 1.909 & 0.16 \\
\hline OCDE/NEA and US NRC & 1a & 1.063792 & 0.6 & 1.682 & 0.04 \\
\hline PWR MOX/UO2 core & $1 c$ & 0.991517 & 1.9 & 2.505 & 0.04 \\
\hline transient & $2 \mathrm{a}$ & 1685.93 & 6.6 & 1.370 & 0.6 \\
\hline $\begin{array}{l}\text { benchmark(Kozlowski } \\
\text { and Downar, 2003) }\end{array}$ & $3 a$ & 1342.93 & 2.2 & 1.748 & 0.0 \\
\hline \multirow{5}{*}{$\begin{array}{l}\text { PWR main steam line } \\
\text { break (MSLB) } \\
\text { benchmarks } \\
\text { (exercise 2)(Ivanov } \\
\text { et al., 1999) }\end{array}$} & 0 & 1.035521 & $182^{\mathrm{a}}$ & 1.3630 & $0.15^{\mathrm{a}}$ \\
\hline & 1 & 1.033542 & $174^{\mathrm{a}}$ & 1.4358 & $0.24^{\mathrm{a}}$ \\
\hline & 2 & 1.004086 & $19^{\mathrm{a}}$ & 1.3293 & $1.79^{\mathrm{a}}$ \\
\hline & 3 & 0.987150 & $175^{\mathrm{a}}$ & 5.4264 & $0.58^{\mathrm{a}}$ \\
\hline & 4 & 1.002067 & $187^{\mathrm{a}}$ & 3.6212 & $0.25^{\mathrm{a}}$ \\
\hline
\end{tabular}

a In this benchmark, reference refers to the average of the solutions given by the different participants.

\subsection{D PWR color-set benchmark}

A straightforward way to test the effect of the transverse leakage (TL) approximation on the nodal solution is to simulate a $2 \mathrm{D}$ color-set problem with two different homogeneous fuel assemblies (FA). Three transverse leakage interpolation schemes are compared, with different nodal refinement degrees. The aim of this comparison is to show that the only source of error in a diffusion solver based on the ACMFD method is in the difference between the real profile of transverse leakage and its interpolation from the nodal averaged interface currents.

Fig. 3 and 4 show the solution in two groups with a set of typical fuel cross-sections and $20 \mathrm{~cm}$ fuel assembly width. The error in both the eigenvalue and the normalized fission source at assembly 1 are analyzed for flat, parabolic and cubic TL interpolation, as a function of the number of meshes in which the assembly is divided. It is considered that ANDES solution with cubic interpolation and $32 \times 32$ meshes per fuel assembly is sufficiently converged and thus taken as the reference solution.

We can see that cubic fit is almost two orders of magnitude more accurate than flat approximation, so the same precision can be achieved taking an eightfold wider mesh. Cubic and parabolic interpolation requires almost the same computing time but, since they lead to similar solutions, not much advantage is found using a cubic fit instead of a parabolic one. The largest convergence rate is for parabolic and cubic fits, mainly for meshes of one or four nodes per assembly, which is the range of interest.

The role of the TL profile in the solution accuracy is determinant. In this test case, the profile is given by the currents at the centreline of the color-set. Figs 5 and 6 show this profile for different fits and nodal refinements $(\times 1, \times 2)$. The $x$ axis goes from the centre of one assembly interface $(-1)$ to the central corner (there is symmetry for the rest of the interface).

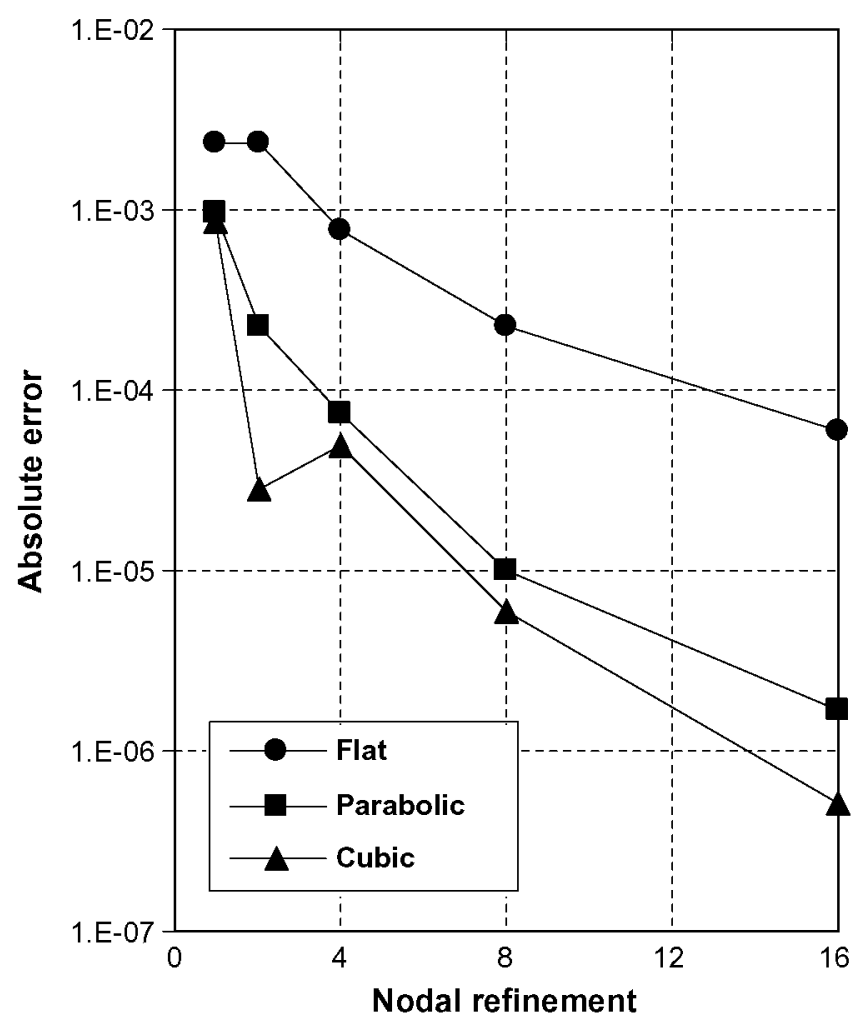

Fig. 3. $K_{\text {eff }}$ absolute error as a function of the nodal refinement respect to converged solution: cubic $(\times 32)$ 


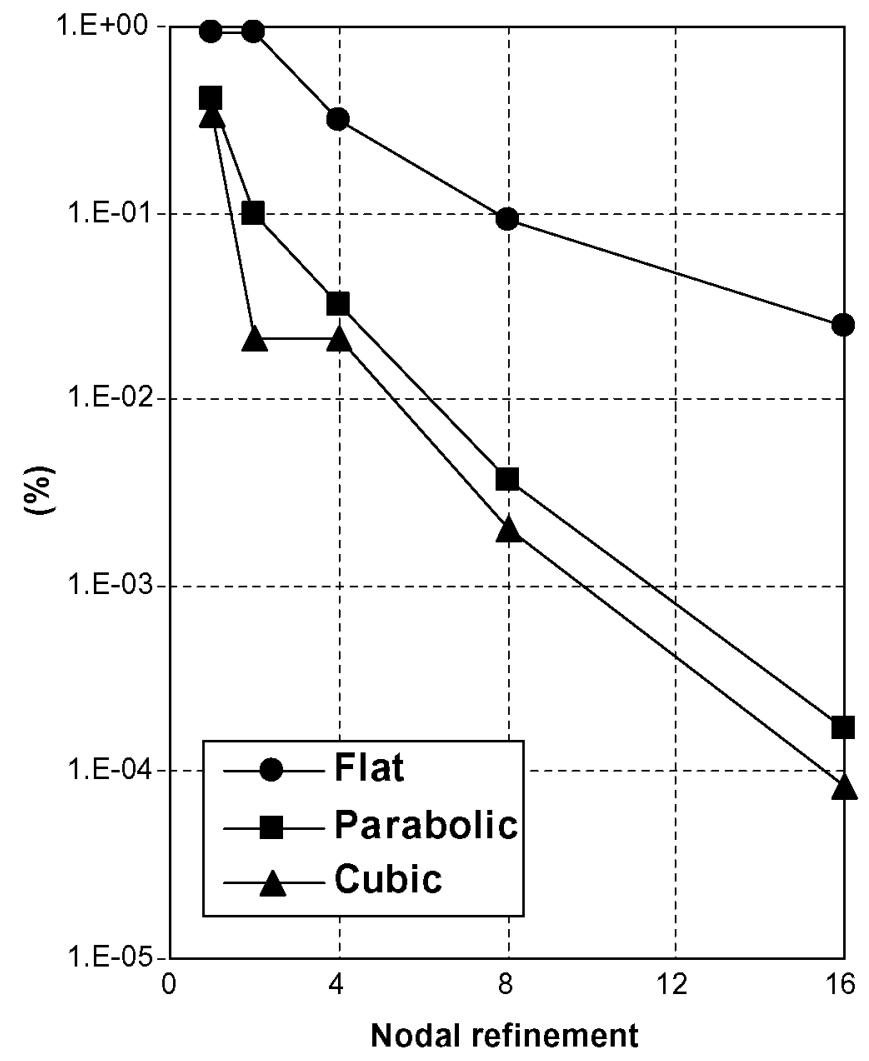

Fig. 4. Fission source relative error $(\%)$ as a function of the nodal refinement respect to converged solution: cubic $(\times 32)$.

\section{COLOR-SET INTERFACE CURRENT PROFILE} (THERMAL GROUP) (x1)

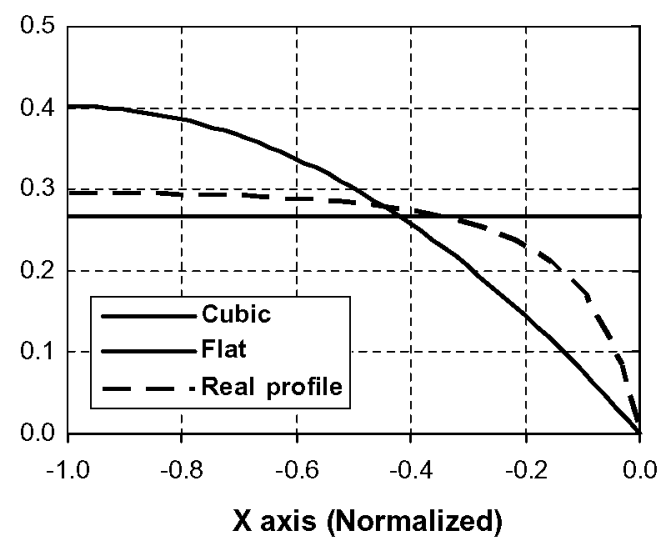

Fig. 5. Thermal current profile for different fits with 1 node per assembly.

It can be seen that the flat approximation overestimates the TL near the assembly edge $(x=0)$ and underestimates them at the assembly centre. When applying the ACMFD relation (6), the ratio between the average fluxes in the two regions is larger than the real value, resulting in a higher $k_{\text {eff. }}$ With the cubic fit, the effect is opposite, so $k_{\text {eff }}$ is underestimated. It is shown that, as stated in Eq. (17), the error in the final solution only depends on the difference between the interpolated TL profile and the real one.
COLOR-SET INTERFACE CURRENT PROFILE (THERMAL GROUP) ( $\times 2$ )

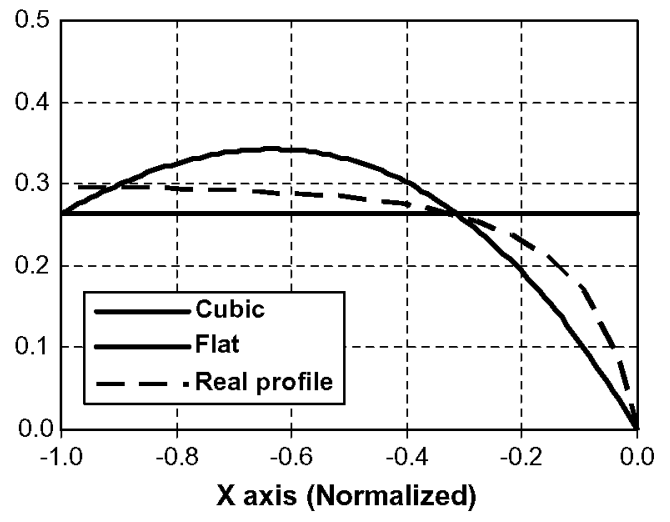

Fig. 6. Thermal current profile for different fits with four nodes per assembly.

\subsection{OECD/NEA and U.S. NRC PWR MOX/UO2 core transient benchmark (Part 3)}

This benchmark allows us to extend the verification of ANDES solver from two up to eight energy groups. It is based on a 3565 MWt nominal power core with 193 UO2/MOX fuel assemblies. Details are described in Ref. (Kozlowski and Downar, 2003).

Part 3 of the benchmark is devoted to characterize the steady state prior to the transient. The core is at HZP state, with the coolant inlet temperature $560 \mathrm{~K}$ and the coolant inlet pressure 15.5 MPa. Control banks are fully inserted while shutdown banks are completely withdrawn. A critical boron concentration search is to be performed.

This steady state has been simulated using the ANDES solver with two, four and eight energy groups. Two different radial coarse-meshes have been tested, with one node per fuel assembly ( $1 \mathrm{~N} / \mathrm{FA}$ ) and with four nodes per fuel assembly ( $4 \mathrm{~N} / \mathrm{FA}$ ), in order to assess the geometry discretization error and the convergence of the results with mesh size. In the axial direction, the core active height is divided into 20 layers, since further subdivision does not modify the solution.

The results obtained with the ANDES solver are compared in Table 2 to those provided by the PARCS code with four nodes per fuel assembly (4 N/FA).

All cases demonstrate good agreement with PARCS, particularly the solutions in two and four energy groups, where differences of 2.2 and $3.2 \mathrm{ppm}$ in critical boron concentration and $0.0 \%$ and $0.07 \%$ in radial peaking factor can be seen.

As an illustrative example, Fig. 7 plots the 3D power distribution computed by ANDES for four nodes/FA and two groups, as obtained using the SALOME graphical post-processing tools.

Table 2

Comparison of critical boron, radial and axial peaking factor between ANDES and PARCS solution

\begin{tabular}{lllll}
\hline & & Critical Boron (ppm) & $F_{X Y}$ & $F_{Z}$ \\
\hline ANDES 2G & $1 \mathrm{~N} / \mathrm{FA}$ & 1343.9 & $\mathbf{1 . 7 4 1}$ & 1.490 \\
& $4 \mathrm{~N} / \mathrm{FA}$ & 1342.9 & $\mathbf{1 . 7 4 8}$ & 1.490 \\
PARCS 2G & $4 \mathrm{~N} / \mathrm{FA}$ & 1340.7 & $\mathbf{1 . 7 4 8}$ & 1.490 \\
ANDES 4G & $1 \mathrm{~N} / \mathrm{FA}$ & 1341.2 & 1.744 & 1.495 \\
& $4 \mathrm{~N} / \mathrm{FA}$ & 1340.2 & $\mathbf{1 . 7 5 1}$ & 1.495 \\
PARCS 4G & $4 \mathrm{~N} / \mathrm{FA}$ & 1337.0 & $\mathbf{1 . 7 5 2}$ & 1.496 \\
ANDES 8G & $1 \mathrm{~N} / \mathrm{FA}$ & 1341.7 & $\mathbf{1 . 7 7 5}$ & 1.496 \\
& $4 \mathrm{~N} / \mathrm{FA}$ & 1340.5 & $\mathbf{1 . 7 8 1}$ & 1.496 \\
PARCS 8G & $4 \mathrm{~N} / \mathrm{FA}$ & 1334.0 & $\mathbf{1 . 7 5 6}$ & 1.498 \\
\hline
\end{tabular}




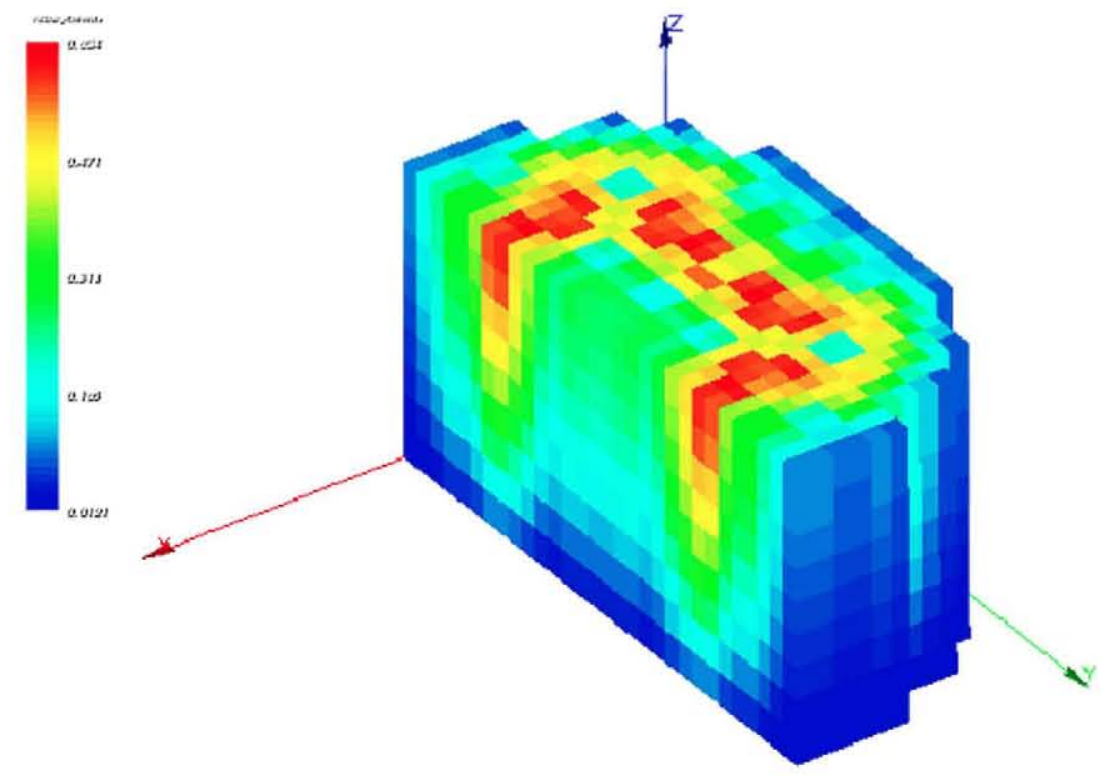

Fig. 7. 3D power distribution computed by ANDES (four nodes/FA and two groups) for the PWR MOX/UO $\mathrm{U}_{2}$ core transient benchmark, part 3.

Up to now, no mention has been done about the convergence of the solution method and computing time. Regarding the method to solve the resulting linear system, the ANDES solver uses an ILU(0) preconditioned $\mathrm{Bi}$-CGSTAB algorithm, which has proved to be robust enough in all range of variability found for the different benchmarks. For the $k_{\text {eff }}$ iterative calculation, the Wielandt method has been implemented, leading to a high reduction in the total number of outer iterations required for convergence (typically $18-20$ iterations to obtain an absolute error of $10^{-8}$ in $k_{\text {eff }}$ and $10^{-3} \%$ of relative error in the fission source).

Fig. 8 shows the evolution of such errors (computed as the difference between two consecutive iterations) during the iterative process for the steady state calculation in eight energy groups. Errors in two and four group solutions are very similar.

Regarding to computing time, Table 3 gives the values for this benchmark exercise, including the time fractions due to the different main tasks. It is to be noted that the ANDES solver is still in an optimization phase, so this issue may be further improved. It can be seen that the fractional time employed computing the eigen-

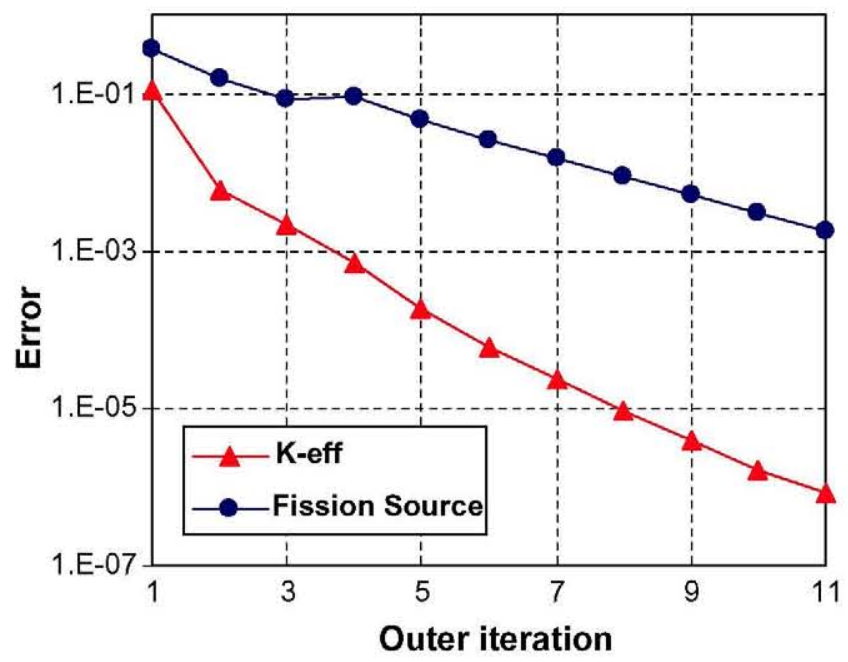

Fig. 8. Evolution of the absolute error both in $k_{\mathrm{e}}$ and fission source along outer iteration for the PWR MOX/UO $\mathrm{U}_{2}$ core transient benchmark, part 3.
Table 3

Computing time for the PWR MOX/UO2 core transient benchmark

\begin{tabular}{|c|c|c|c|c|c|c|}
\hline & \multicolumn{2}{|l|}{$2 G$} & \multicolumn{2}{|l|}{$4 G$} & \multicolumn{2}{|l|}{$8 G$} \\
\hline & $1 \times 1$ & $2 \times 2$ & $1 \times 1$ & $2 \times 2$ & $1 \times 1$ & $2 \times 2$ \\
\hline Total time (s) & 4.393 & 20.303 & 8.934 & 40.005 & 20.994 & 88.30 \\
\hline $\begin{array}{l}\text { Relative time to the } 2 G \\
1 \times 1 \text { case }\end{array}$ & 1.0 & 4.6 & 2.0 & 9.1 & 4.8 & 20.1 \\
\hline $\begin{array}{l}\text { Eigenvalues and } \\
\text { eigenvectors (\%) }\end{array}$ & 14.4 & 12.7 & 20.1 & 17.5 & 23.8 & 22.5 \\
\hline $\begin{array}{l}\text { Linear system } \\
\text { coefficients (\%) }\end{array}$ & 34.9 & 32.2 & 33.6 & 32.1 & 35.0 & 33.0 \\
\hline Preconditioning (\%) & 25.3 & 21.7 & 24.6 & 21.7 & 27.5 & 25.0 \\
\hline Bi-CGSTAB (\%) & 22.9 & 30.9 & 20.3 & 27.3 & 12.9 & 18.8 \\
\hline Interface currents (\%) & 2.6 & 2.5 & 1.5 & 1.4 & 0.8 & 0.8 \\
\hline
\end{tabular}

This case has been run under linux, in a Pentium $43.2 \mathrm{GHz}$.

values and eigenvectors of the multigroup diffusion matrix becomes more important as the number of energy groups increases. On the other hand, the fractional time spent in solving the linear system (Bi-CGSTAB) decreases with the number of groups, but increases with the number of nodes per assembly. Preconditioning fractional time importance remains quite stable but shows a trend to decrease with the number of nodes. With regard to the total time, and taking into account that the unknowns of the linear system are the nodal average fluxes per energy group, we can see that computing time is almost proportional to the total number of unknowns.

\subsection{Rod "cusping" benchmark problem}

In order to assess the methodology developed in ANDES to reduce the effect of rod "cusping", a very simple 3D benchmark has been designed. This benchmark consists of a $3 \times 3$ identical fuel assembly core where a control rod can be inserted in the central assembly. Each assembly has a radial dimension of $21.42 \times 21.42 \mathrm{~cm}$. Radially, reflective boundary conditions are imposed. Axially, the active core height is $200 \mathrm{~cm}$, and the core is lower and upper reflected by $20 \mathrm{~cm}$ of water. Cross-sections have been taken from the PWR/MOX benchmark two-group library with fixed thermal-hydraulic conditions. 

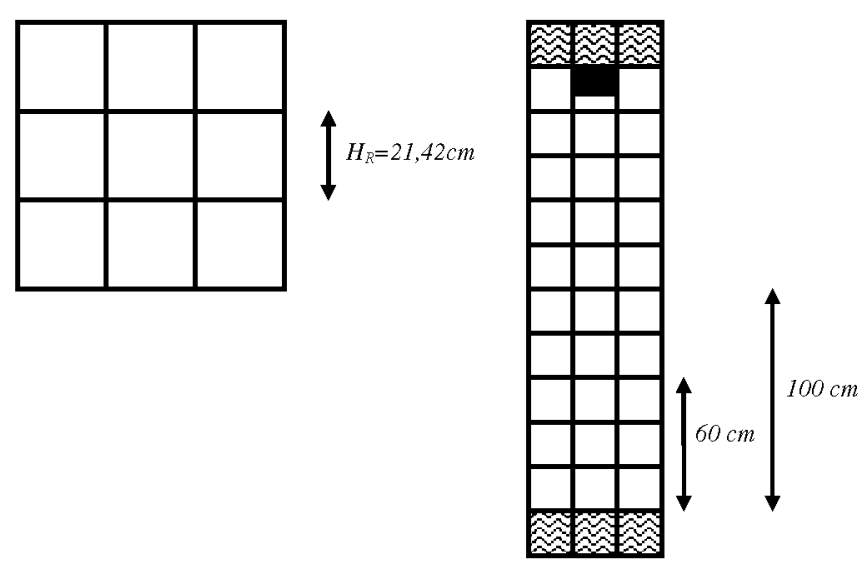

Fig. 9. Coarse-mesh nodalization for the rod "cusping" benchmark problem.

A sequence of eigenvalue calculations are performed for different control rod insertions, going from 100 to $60 \mathrm{~cm}$. This way, the rod "cusping" effect can be observed in $k_{\text {eff }}$ as the control rod tip goes through two nodes.

The coarse-mesh nodalizations used in ANDES are illustrated in Fig. 9. Radially, one node per assembly is taken. In the axial direction, the core is divided into 12 equally meshes of $20 \mathrm{~cm}$, including reflectors. To provide a reference solution, a second axial nodalization is implemented, subdividing the two axial meshes where the control rod is going to be partially inserted in eight axial meshes each one of $H_{Z}=2,5 \mathrm{~cm}$. Finally, to evaluate the rod "cusping" correction, the low-order approach for treating the rod "cusping" problem is also simulated. This approach consists of a parabolic interpolation of the axial fluxes to obtain the average fluxes needed to weight the rodded and unrodded cross-sections in Eq. (23).

Fig. 10 shows the results in $k_{\text {eff }}$ of the two methods (ANDES and low-order) compared with the reference solution mentioned in the previous paragraph. Nodes of 20 and $10 \mathrm{~cm}$ have been compared in both cases. The most important conclusion is that the correction implemented in the ANDES solver reduces the maximum absolute error in $k_{\text {eff }}$ by a factor of five, leading to similar reductions in power oscillations during control rod transients. Besides, if the axial node size is reduced to $10 \mathrm{~cm}$, while the low-order approach divides the error by three, in the analytic procedure in ANDES the error is divided by six. Finally it is worthy to remark that the $k_{\text {eff }}$

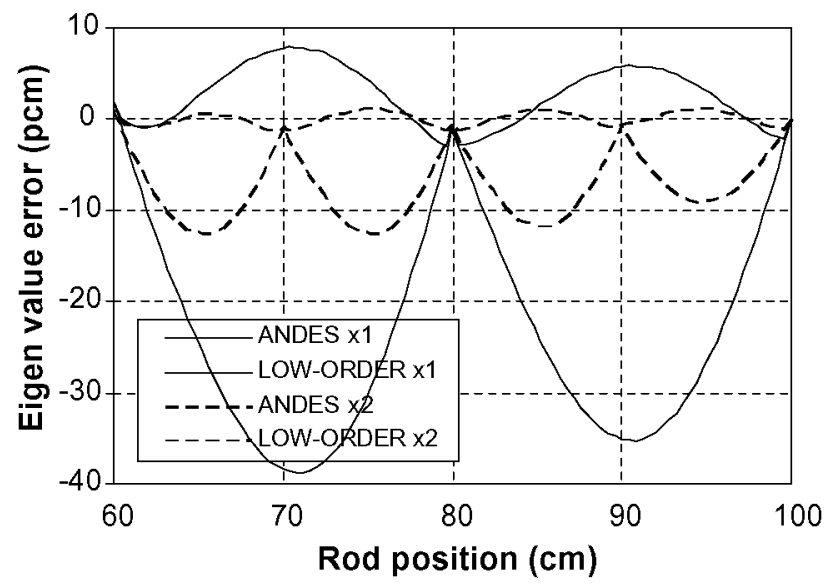

Fig. 10. Eigenvalue error obtained with ANDES and the low-order approach (fluxvolume weighting) for an axial mesh of $10 \mathrm{~cm}(\times 2)$ and $20 \mathrm{~cm}(\times 1)$. deviation with the new approach has the opposite sign than the low-order one, since the interpolation of the radial currents reduces the leakage of the unrodded part of the node and, consequently, overestimates the average flux in this region, resulting in a larger $k_{\infty}$ for the node.

\section{Summary}

The analytic nodal diffusion extended solver ANDES has been developed extending the ACMFD method from two-group and two dimensions to any number of groups and three dimensions in rectangular geometry. Non-linear schemes for interpolation of the transverse leakage terms in the ACMFD method have been implemented, exhibiting high accuracy and robust and fast convergence. The solver has been implemented in a fully encapsulated way, enabling it as a module to be readily integrated in other codes and platforms. Verification of performance has shown that ANDES is a code with high accuracy, both in neutron energy (any number of groups) and spatial intranodal distribution (even for heterogeneous large nodes), while the computing time is kept proportional to the number of unknowns (nodes $\times$ groups). The efficient extension of the analytic method to nodes with control rods partially inserted, to solve the rod "cusping" problem, is a paradigm of the advantages of the ACMFD method for whole core realistic nodal simulations.

The set of tests and benchmarks done for ANDES verification have demonstrated its high convergence rates, in terms of mesh refinement, thanks to the ACMFD method implemented. Using $1 \times 1$ nodes per PWR fuel assembly, the $k_{\text {eff }}$ absolute error obtained is below $50 \mathrm{pcm}$ and the maximum assembly fission source error is below $1 \%$. Using $2 \times 2$ nodes per PWR assembly, which is the reference/recommended node size, the obtained errors reduce to $2 \mathrm{pcm}$ and $0.2 \%$, respectively, a level of accuracy such that a standard finemesh finite-difference scheme would need $136 \times 136$ cells per assembly to achieve. It is convenient to remark that for this analysis the same nodal cross-sections are used in nodes within each assembly as the radial mesh is refined.

The ongoing work in the framework of ANDES includes the development, implementation and verification of additional computational capabilities and improvements, in process of advanced verification, namely:

- Extension of the ACMFD method to time dependent 3D neutron kinetics coupled to thermal-hydraulics for efficient and accurate nodal simulation of fast transients in anticipated operational occurrences and design-basis accidents.

- Extension of the ACMFD method to 3D triangular- $Z$ geometry to allow the simulation of hexagonal lattice fuel assemblies and cores in VVER, HTR and fast reactors.

- Development and implementation of analytical multigroup response matrices, consistent with the ACMFD method, to replace the radial and axial reflectors by generalized boundary conditions at the core-reflector interfaces, independent of the core loading patterns and cycle burnups.

\section{Acknowledgments}

This work is part of the PhD Thesis of the first author, which is tutored by the other authors, being partially funded by the EC Commission under the 6th EURATOM Framework Programme, within the RTD Integrated Project NURESIM "European Platform for Nuclear Reactor Simulations", Contract No. 516560 (FI6O). It is also funded by the Universidad Politécnica de Madrid and MEC within the program ENE2005-02064/FTN. 


\section{References}

Aragonés, J.M., Ahnert, C., García-Herranz, N., 2007. The analytic coarse-mesh finite difference method for multigroup and multidimensional diffusion calculations. Nucl. Sci. Eng. 157, 1-15.

Cacuci, D.G Aragonés, JM., Bestion, D Coddington, P., Dada L Chauliac, C, 2006. NURESIM: A European Platform for Nuclear Reactor Simulation. Invited paper in FISA 2006 - EU Research and Training in Reactor Systems, Luxembourg, pp. 123-143.

Calvin, C., 2005. DESCARTES: a new generation system for neutronic calculations. In: Proc. Int. Conf. on Mathematics and Computation, M\&C-2005, Avignon, France.

Chao, Y.A., 1999. A Theoretical Analysis of the Coarse Mesh Finite Difference Representation in Advanced Nodal Methods. In: Aragonés, J.M. (Ed.), Mathematics and Computation, Reactor Physics and Environmental Analysis in Nuclear Applications, vol. 1. Senda Ed., Madrid, pp. 117-126.

Chao, Y.A., 2000. Coarse mesh finite difference methods and applications. In: Proc. lnt. Topl. Mtg. Advances in Reactor Physics and Mathematics and Computation into the Next Millennium, PHYSOR-2000, vol.1, 9404, American Nuclear Society, Pittsburgh.

Finnemann, H., Bennewitz, F., Wagner, M.R., 1977. Interface current techniques for multi-dimensional reactor calculations. Atomkernenergie 30, 123.

Finnemann, H., Galati, A., 1991. NEACRP 3D LWR Core Transient Benchmarks, Final Specifications. OECD/NEACRP-L-335.

Fraikin, R., 1997. PWR Benchmark on Uncontrolled Rods Withdrawal at Zero Power. Final Report, NEA/NSC/DOC(96) 20.

García-Herranz, N. Cabellos, O., Aragonés, J.M. Ahnett, C, 2003. Analytic coarse mesh finite difference method generalized for heterogeneous multidimensional two-group diffusion calculations. Nucl. Sci. Eng. 144, 23-35.

Herrero, J.]., Ahnert, C., Aragonés, J.M., 2007. 3D whole core fine mesh multigroup diffusion calculations by domain decomposition through alternate dissections.
In: Rubia, T., Vujic, J. (Eds.), Mathematics and Computations and Supercomputing in Nuclear Applications, M\&C/SNA-2007 Monterey CA, vol. 8D9, 1-13, American Nuclear Society Ed., La Grange Park, IL-USA, ISBN: 0 89448-059-6.

Ivanov, K.N., Beam, T.M., Irani, A., Trikouros, N., 1999. Pressurized Water Reactor Main Steam Line Break (MSLB) Benchmark. volume I: Final Specs, NEA/NSC) $\operatorname{DOC}(99) 8$.

Joo, H.G., Barber, D.A., Jiang, G., Downar, T.J., 1998. PARCS: A Multi-Dimensiona Two-Group Reactor Kinetics Code Based on the Nonlinear Analytic Nodal Method. Purdue University. PU/NE-98-26.

Kozlowski, T., Downar, T., 2003. OECD/NEA and US NRC PWR MOX/UO $\mathrm{U}_{2}$ core transient benchmark, Final Specifications, Revision 2. NEA/NSC/DOC(2003)20.

Müller, E.Z., Wiederhold, A.F., 1995. Selected non-linear iterative schemes for the multigroup analytic nodal method. In: Int. Conf. on Math. and Comp., Reactor Physics and Env. Anal., Portland, Oregon, USA.

Noh, J.M., Cho, N.Z., 1994. A new approach of analytic basis function expansion to neutron diffusion nodal calculations. Nucl. Sci. Eng. 116, 165.

Smith, K.S., 1979. An analytic nodal method for solving the two-group multidimensional, static and transient neutron diffusion equations. Nucl. Eng. Thesis, Department of Nuclear Engineering, MIT, Cambridge, MA.

Smith, K.S., 1986. Assembly homogenization techniques for light water reacto analysis. Prog. Nucl. Energ 17 (3), 303-355.

Turinsky, F.J., Sarour, H.N., Al-Chalabi, R.M.K., Engrand, P., Faure, F.X., Guo, W., 1994 NESTLE: A Few-Group Neutron Diffusion Equation Solver Utilizing the Nodal Expansion Method for Eigenvalue, Adjoint, Fixed-Source Steady-State and Transient Problems. EGG-NRE-11406, INEL.

Vogel, D.L., Weiss, Z.I., 1992. A general multigroup formulation of the analytic nodal method. In: Proc. Int. Topical Meeting on Advances in Reactor Physics, Charleston, South Carolina, USA.

Ziyong, L., Shaohong, Z., Chao, Y.A., 2006. A theoretical study on a convergence problem of nodal methods. In: PHYSOR-2006, Vancouver. 